\title{
High Temperature Resonant Ultrasound Spectroscopy: A Review
}

\author{
G. Li and J. R. Gladden \\ Department of Physics and Astronomy, National Center for Physical Acoustics, The University of Mississippi, MS 38677, USA
}

Correspondence should be addressed to J. R. Gladden, jgladden@olemiss.edu

Received 6 August 2010; Accepted 24 November 2010

Academic Editor: Jaan Laane

Copyright ( $) 2010$ G. Li and J. R. Gladden. This is an open access article distributed under the Creative Commons Attribution License, which permits unrestricted use, distribution, and reproduction in any medium, provided the original work is properly cited.

The measurement of elastic constants plays an important role in condensed matter physics and materials characterization. This paper presents the resonant ultrasound spectroscopy (RUS) method for the determination of elastic constants in a single crystal or amorphous solid. In RUS, the measured resonance spectrum of a properly prepared sample and other information such as geometry, density, and initial estimated elastic constants are used to determine the elastic constants of the material. We briefly present the theoretical background and applications to specific materials; however, the focus of this review is on the technical applications of RUS, especially those for high-temperature measurements.

\section{Introduction: Elastic Constants and Measurement Methods}

The elastic response of a solid is determined by the full set of independent elastic constants, which are a measure of the material's interatomic forces and, specifically, the curvature of the potentials around the equilibrium spacing. Elastic constants are a sensitive probe into the atomic environment of a crystal lattice, and changes in elastic constants are a useful tool for investigating critical phenomenon. Elastic constants are involved in many fundamental phenomena in solid-state physics: they are important parameters in equations of state, lattice dynamics, and phonon spectra; they are also linked to other quantities in thermodynamics such as coefficient of thermal expansion, Debye temperature, Grüneisen parameter, and so on. The measurement of elastic constants is of interest not only to engineers and materials scientists, but also to researchers in many areas of fundamental and applied physics.

Numerous theoretical and experimental methods are available for evaluating elastic constants. If the equation for the interatomic potential is known, the elastic constant can be calculated from first principles. The results from ab initio calculations for some crystalline solids with known atomic structures and potentials are usually in reasonable agreement with the experimental data. For simple, accurate and efficient determination of elastic properties of materials, various ultrasonic and nonultrasonic experimental techniques are often preferred.

Many experimental techniques [1-3] have been developed and employed for measuring the elastic constants of different types of materials. The selection of technique depends on factors such as the composition, structural characteristic and size of the sample, desired accuracy of measurement, and, of course, the availability of equipment and expertise. Various common techniques can be roughly categorized according to the major parameters that are evaluated or the primary equipment that are used (Table 1). The accuracy of a given experimental method depends on many factors other than the fundamental nature of the method itself. However, frequencies are one of the easiest quantities to measure, and resonance methods typically depend on many more frequency measurements than variables being determined. For these reasons, RUS has emerged as one of the most accurate methods for elastic constant measurements.

In principle, elastic constants can be readily computed from the stress and strain, which are related by the generalized Hooke's law throughout the elastic range. Samples of relatively large sizes are either pulled, compressed, bent, or twisted by known stresses, and the strains are measured by a variety of techniques such displacement measurement 
TABLE 1: Comparison of techniques commonly used for elasticity evaluation.

\begin{tabular}{ll}
\hline $\begin{array}{l}\text { Main parameters } \\
\text { measured/computed }\end{array}$ & Representative methods \\
\hline Theoretical/numerical & Ab initio calculation \\
Stress-strain & Static, quasi-static, indentation \\
Speed of sound & Pulse-echo, continuous wave \\
Frequencies & Resonance, RUS \\
Diffraction/Scattering & X-ray, neutron, Brillouin \\
\hline
\end{tabular}

by a strain gauge. When a sample can be conveniently put into some kind of vibration, a quasistatic method is often preferred over the static method in strain and stress measurements. In the quasistatic method, the sample is subject to a small, harmonic external force, and frequency of the external force is much lower than the natural resonance frequencies of the sample itself. Both static and quasi-static stress-strain methods are widely used for characterizing materials for engineering applications. It is important to note that because the time scales of static and quasi-static methods are typically long compared to thermal time scales, the sample has time to come to thermal equilibrium in response to the stress, and thus the moduli obtained are isothermal moduli. This is in contrast to the much shorter time scales in ultrasonic measurements which produce adiabtic moduli.

New and exotic materials of crystal forms are often small, and the number of independent elastic constants increases as crystalline symmetry decreases. Due to their high efficiency and accuracy, ultrasonic methods are better suited to evaluating elastic properties of these materials. Ultrasonically, an elastic constant can be measured in two ways: one is by measuring the speed of sound, which is related to the relevant elastic constant component; the other is by measuring the free vibration frequencies, which depend on sample density, dimensions, and elastic constants. Elastic constants can be determined from the ultrasonic wave velocities in various directions in a crystal [4]. In the linear regime, the general relationship between the speed of sound in a crystal, the material density, and its elastic constants is governed by the following equation:

$$
v=\sqrt{\frac{C_{i j}}{\rho}}
$$

where $v$ is the speed of sound, $C_{i j}$ is the component of the elastic constants relevant to $v$ (i.e., for a shear wave, $C_{44}$ would be the relevant constant), and $\rho$ is the material density. The pulse-echo method is the most commonly used method of measuring speed of sound in homogeneous and anisotropic materials $[4,5]$. In the pulse-echo method, good acoustic coupling or even bonding between the sample and transducer is required. To obtain all the elastic constants of a crystal, the same sample must be repeatedly cut along different principal axes of the crystal lattice and bonded to the transducer, or multiple samples are needed. This is rather labor intensive, especially for low-symmetry crystals. The lack of suitable high-temperature piezoelectric transducers

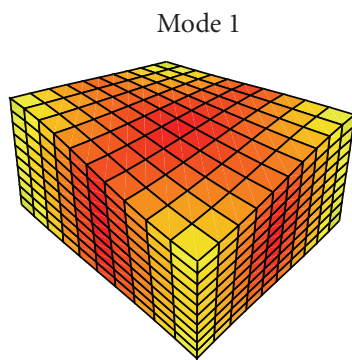

(a)

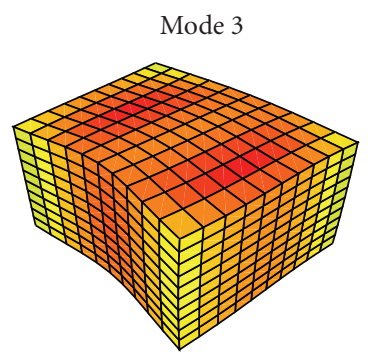

(c)

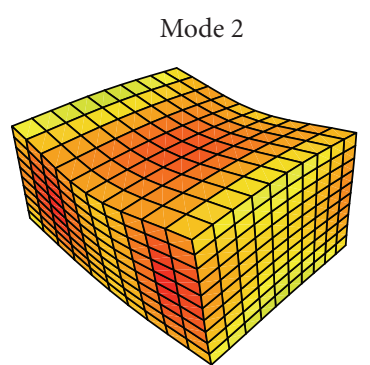

(b)

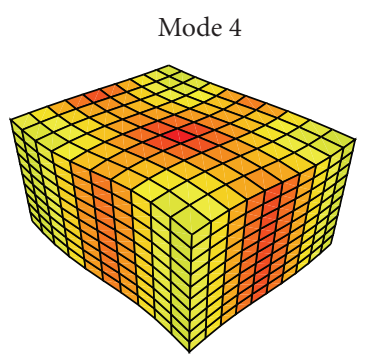

(d)
Figure 1: (Color online) Several lower normal modes of a parallelepiped sample. Note that surface amplitudes are exaggerated for clarity. Darker (red in color) areas denote nodal regions, and lighter (yellow in color) areas denote antinodal regions.

or stable high-temperature couplants prevents the pulseecho method from being a robust method for elastic constant measurement in such extreme environments.

Resonance methods have been useful tools for measuring elastic properties of solid materials for many years. An elastic body resonates when the frequency of the external force matches one of the natural frequencies of the body. The response of the body during resonance is amplified by roughly the quality factor $\left(Q \approx f_{0} / \Delta f\right.$, where $\Delta f$ is the peak width at half magnitude) and can be easily detected by piezoelectric transducers. Several lower normal modes for a particular parallelepiped sample are demonstrated in Figure 1. These free-vibration frequencies, along with dimensions and mass of the sample, can be used to infer the elastic properties of the material. This is the so-called "inverse" problem-inferring elastic constants from measured free-vibration frequencies. The solution of the inverse problem relies on solving the "forward" problem first, that is, to compute its normal modes from the known characteristics of an elastic body. Early resonance techniques were largely confined to large-sized samples with regular geometries such as a sphere for which analytical solutions for the associated forward resonance problems were available [6-8]. The lack of exact solutions to the problem of the free vibrations of an elastic solid of general shape and crystallographic symmetry greatly limited the scope of resonance methods until highspeed computers became common.

Early researchers $[9,10]$ attempted to use the RayleighRitz variational technique [11] to calculate the natural vibration frequencies of parallelepipeds. Demarest [12] developed a more accurate method called "cube resonance" for solving 
the forward problem using the Rayleigh-Ritz method, and this method was used for elastic constant determination with limited success. The cube resonance method was further extended by Ohno [13] to the determination of elastic constants of single crystals. Ohno called his method "rectangular parallelepiped resonance" and it is abbreviated as RPR. Subsequently, researchers advanced the RPR method and applied it to the determination of elastic constants of solids [14-25].

When Migliori and Maynard examined the history of the modern resonant ultrasound spectroscopy (RUS) method [26], they found that a milestone in RUS was the identification of a simple set of basis functions - the products of powers of the Cartesian coordinates by Visscher et al. [27]. This set of basis functions can be applied to many geometries and general anisotropic materials with great accuracy and flexibility. With this breakthrough in solving the forward problem and the wide availability of computing resources, RUS was soon accepted as a useful technique for determining elastic constants of materials, particularly small crystalline solids [2, 28]. RUS methods have been refined and extended over the past several decades to include measurements in extremely low and high temperatures, high pressures, high magnetic fields, as well as heterogeneous samples composed of thin films deposited on a substrate [13, 18, 26, 28-32].

\section{Theoretical Method}

Two problems, a "forward" and an "inverse" problem, are involved in computing (estimating to be exact) the elastic constants of a solid from the measured spectrum. The forward problem involves computing the spectrum from known parameters of dimensions, density, and initial estimated values for elastic constants; the so-called inverse problem involves determining all independent elastic constants from the experimentally obtained spectrum. The forward problem is presented first.

2.1. Linear Elasticity Theory Primer. The linear theory of elasticity has been well treated in detail in many classical textbooks [33-36]. When the body of a solid material is deformed under external forces, a point $\mathbf{r}$ (with components $x_{i}$ ) in the undistorted body moves to a new location $\mathbf{r}^{\prime}$ (with components $x_{i}^{\prime}$ ). The displacement of this point due to the deformation is then given by the vector $\psi=\mathbf{r}^{\prime}-\mathbf{r}$, which we shall denote by

$$
\psi_{i}=x_{i}^{\prime}-x_{i}
$$

The $\psi$ is called the displacement vector. Since the vector $\mathbf{x}^{\prime}$ is itself a function of $\mathbf{x}$, this implies that a small deformation of an elastic body can be written as

$$
d \psi_{i}=\psi_{i, 1} d x_{1}+\psi_{i, 2} d x_{2}+\psi_{i, 3} d x_{3}=\psi_{i, j} d x_{j}
$$

where the second subscript denotes a partial derivative has been performed with respect to the direction indicated

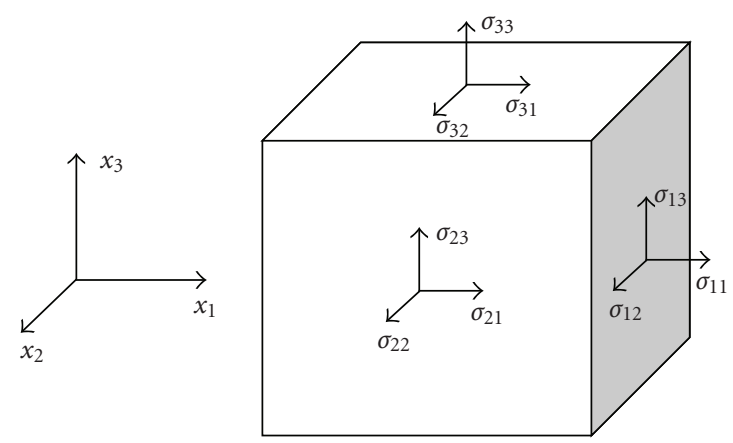

Figure 2: Components of stress tensor acting on a small element. For a stress component $\sigma_{i j}$, the first index $i$ indicates the plane that the stress acts on, and the second index $j$ denotes the direction in which the stress acts.

(i.e., $\left.\psi_{i, 1}=\partial \psi_{i} / \partial x_{1}\right)$. Rewrite (3) into symmetrical and antisymmetric components

$$
d \psi_{i}=\frac{1}{2}\left(\psi_{i, j}+\psi_{j, i}\right)+\frac{1}{2}\left(\psi_{i, j}-\psi_{j, i}\right),
$$

where the Einstein summation convention (summation over repeated indices) is used here. The second term represents a solid body rotation, which is independent of any elastic deformation so it can be dropped. We define the strain as

$$
\epsilon_{i j}=\frac{1}{2}\left(\psi_{i, j}+\psi_{j, i}\right)
$$

where it is assumed that the strains are small so that secondorder terms may be neglected. A strain tensor represents the distortion produced in the body by the applied forces. Note that the strain tensor is symmetric

$$
\epsilon_{i j}=\epsilon_{j i}
$$

so there are only six independent components. The stress is a measure of the intensity of total internal forces when the body of a solid material is deformed. The components of the forces applied to an imaginary surface inside a solid can be written as

$$
d t_{i}=\sigma_{i j} d S_{j}
$$

$d t_{i}$ are the components of the forces acting on the small surface of $d S_{j} ; \sigma_{i j}$ is the stress tensor having units of force per unit area. The components of stress tensor acting on a small element are demonstrated in Figure 2. For a solid in static equilibrium, the net torque acting upon the body is zero, requiring the stress tensor to be symmetrical, that is,

$$
\sigma_{i j}=\sigma_{j i}
$$

Similar to the strain tensor, there are only six independent components in the stress tensor.

In linear elasticity theory, each stress component can be expressed as a linear combination of the strain components. The stress and the strain are related by the generalized Hooke's law

$$
\sigma_{i j}=c_{i j k l} \epsilon_{k l}
$$


TABLE 2: Independent elastic constants for various crystal symmetries.

\begin{tabular}{lcl}
\hline Crystal class & $\begin{array}{c}\text { Number } \\
\text { of } C_{i j}\end{array}$ & List of elastic constants \\
\hline Triclinic & 21 & All possible combinations \\
Monoclinic & 13 & $\begin{array}{l}C_{11} ; C_{12} ; C_{13} ; C_{16} ; C_{22} ; C_{23} ; C_{26} ; \\
C_{33} ; C_{36} ; C_{44} ; C_{45} ; C_{55} ; C_{66}\end{array}$ \\
Orthorhombic & 9 & $C_{11} ; C_{12} ; C_{13} ; C_{22} ; C_{23} ; C_{33} ; C_{44} ;$ \\
Trigonal & 6 or 7 & $C_{55} ; C_{66}$ \\
Tetragonal & 6 & $C_{11} ; C_{33} ; C_{44} ; C_{13} ; C_{12} ; C_{14} ; C_{25} ; C_{33} ; C_{44} ; C_{13} ; C_{12} ; C_{66}$ \\
Hexagonal & 5 & $C_{11} ; C_{33} ; C_{44} ; C_{12} ; C_{14}$ \\
Cubic & 3 & $C_{11} ; C_{12} ; C_{44}$ \\
Isotropic & 2 & $C_{11} ; C_{44}$ \\
\hline
\end{tabular}

where $c_{i j k l}$ are components of the elastic stiffness tensor. The symmetries in the strain and the stress reduce 81 different entries in the elastic tensor 36 independent elements. In the linear regime, the potential energy must be quadratic in the strain. This reduces the number of independent elements to 21 . This number can be further reduced by the crystallographic symmetries imposed by the material itself. In the literature, the elastic constants are often written in the reduced index form. Voigt notation is the standard mapping for tensor indexes $(11 \rightarrow 1 ; 22 \rightarrow 2 ; 33 \rightarrow$ $3 ; 23,32 \rightarrow 4 ; 13,31 \rightarrow 4 ; 12,21 \rightarrow 6)$. The cubic system is the simplest anisotropic system, there are only three independent elastic constants $\left(C_{11}, C_{12}\right.$, and $\left.C_{44}\right)$. Note we use a lowercase $c$ when denoting the full fourth rank stiffness tensor and capital $C$ when discussing the reduced notation form. Isotropic materials have only two independent elastic constants, which are usually expressed as the first-order elastic moduli: Young's modulus (E) and Poisson's ratio $(\nu)$. However, the alternative elastic constants bulk modulus (K) and/or shear modulus $(\mathrm{G})$ can also be used. Table 2 lists the arrays of reduced elastic constants for various crystal symmetries.

2.2. Computation of Resonance Frequencies. The calculation of vibration modes of elastic objects with free boundaries is a classic problem in mechanics. Unfortunately, exact analytical solutions exist for only a few cases such as an isotropic sphere and certain modes of a parallelepiped [7]. In most cases, approximation methods such as finite element method (FEM) or Rayleigh-Ritz method must be used for estimating the eigenfrequencies of normal modes. FEM allows the determination of eigenfrequencies for an arbitrary shape of sample made of heterogeneous, anisotropic materials such as layered composite materials. FEM is more general and usually requires more computing time [37]. The RayleighRitz method [11] is a widely used, classic method for calculating the approximate natural vibration frequencies of a system. The application of the Rayleigh-Ritz method in RUS calculation is based on the Hamilton's principle of least action that the Lagrangian of a system is stationary with respect to small perturbations in the eigenfunctions. The potential and kinetic energy can be expressed in terms of a displacement field $(\psi)$. The potential energy density is

$$
U=\frac{1}{2} c_{i j k l} \epsilon_{i j} \epsilon_{k l}=\frac{1}{2} c_{i j k l} \frac{\partial \psi_{i}}{\partial x_{j}} \frac{\partial \psi_{k}}{\partial x_{l}}=\frac{1}{2} c_{i j k l} \psi_{i, j} \psi_{k, l},
$$

and the kinetic energy density for a normal mode is

$$
T=\frac{1}{2} \rho \omega^{2} \psi_{i} \psi_{i}
$$

where an $\exp (i \omega t)$ time dependence has been assumed and first derivatives with respect to time have been performed $(v=d \psi / d t)$. The Lagrangian is defined as $L=T-U$. According to the Hamilton's principle, the time integral of the Lagrangian is a constant of motion, that is

$$
\delta \int_{t 1}^{t 2} \int_{V}(T-U) d V d t=0 .
$$

The displacement field can be approximated by an expansion of some suitable set of basis functions:

$$
\psi_{i}(\vec{r}) \approx a_{i \alpha} \Phi_{\alpha}(\vec{r}),
$$

where $\alpha=(l, m, n)$ is a set of nonnegative integers. Substituting the expression above into the Lagrangian, we get

$$
\begin{aligned}
L= & T-U \approx \frac{1}{2}\left(a_{i \alpha} a_{i^{\prime} \alpha^{\prime}} \rho \omega^{2}\right) \int_{V} \delta_{i i^{\prime}} \Phi_{\alpha}(\vec{r}) \Phi_{\alpha^{\prime}}(\vec{r}) d V \\
& -\frac{1}{2} a_{i \alpha} a_{i^{\prime} \alpha^{\prime}} \int_{V} c_{i j i^{\prime} j^{\prime}} \Phi_{\alpha, j}(\vec{r}) \Phi_{\alpha^{\prime}, j^{\prime}}(\vec{r}) d V .
\end{aligned}
$$

Writing the above compactly in the matrix format:

$$
L=\frac{1}{2}\left(\left(\rho \omega^{2}\right) a^{T} \mathbf{E} a-a^{T} \mathbf{\Gamma} a\right)
$$

where both $\mathbf{E}$ and $\Gamma$ are matrices, and have elements, respectively,

$$
\begin{gathered}
E_{\alpha i \alpha^{\prime} i^{\prime}}=\delta_{i i^{\prime}} \int_{V} \Phi_{\alpha} \rho \Phi_{\alpha^{\prime}} d V \\
\Gamma_{\alpha i \alpha^{\prime} i^{\prime}}=c_{i j i^{\prime} j^{\prime} j^{\prime}} \int_{V} \Phi_{\alpha, j} \Phi_{\alpha^{\prime}, j^{\prime}} d V .
\end{gathered}
$$

The requirement that Lagrangian is stationary, that is, the variations of Lagrangian with respect to a (any combination of a) is zero, allows us to cast the problem in the matrix eigenvalue form:

$$
\Gamma \mathbf{a}=\left(\rho \omega^{2}\right) \mathbf{E} \mathbf{a} .
$$

The components of the vector a are the expansion coefficients, and the eigenvalues $\omega$ correspond to the square of the angular frequencies of normal modes.

The central part of RUS calculation consists of solving (18) (finding the set of a which minimizes the Lagrangian $L$ ) and then using the computed eigenvalues to "correct" the set of input parameters so as to iterate toward a match between the computed and measured frequencies (eigenvalues). There are standard computer routines for solving 
such eigenvalue problems. Any computation involving large matrix (typically $1000 \times 1000$ in RUS) is always time consuming. The symmetries in the sample geometry and elastic constant matrix can be exploited to split large matrices through block diagonalization into smaller ones, and each can be solved independently with significant savings in computation time [13]. A good set of basis functions to approximate the displacement field is important for the RUS calculation. Choice of basis functions and estimation of elastic constants are briefly addressed in the following two sections.

2.3. Choice of Basis Functions. Many functions such as trigonometric functions and Legendre polynomials have been exploited as the basis functions. They are simple, orthogonal, and complete functions but they lack the flexibility to handle a large number of sample shapes. Polynomials are good candidates for basis functions because a simple standard analytical solution exists for the integrals and derivatives of the polynomials, and high-order polynomials can approximate many shapes.

Visscher et al. proposed a simple set of basis functions in a paper published in 1991 [27]: the simple powers of the Cartesian coordinates,

$$
\Phi_{\alpha}=x^{p} y^{q} z^{r}
$$

where $(p, q, r)$ are a set of positive integers indexed by $\alpha$ with the maximum power set by $N \leq p+q+r$. This is known as the $\mathrm{XYZ}$ algorithm, and it is applicable to various irregularshaped and anisotropic materials. A typical number of 10 for $N$ is numerically adequate to approximate the actual functions and very flexible and convenient to implement for a variety of shapes. The larger the $N$, of course, the more accurately the basis functions mimic the actual normal mode displacements. However, a higher power of $N$ does not necessarily yield a better solution. The increased demand in computing power and the digital precision of the computer processors must be considered. Typically, $N=13$ is the limit for a 32-bit computer. A further benefit of an expansion of this form is that integrals and derivatives of this function have the same functional form, only changing the coefficients and powers in a simple way.

2.4. Estimation of Elastic Moduli. The determination of the elastic constants from the measured resonance frequencies is the inverse of the forward problem. The forward calculation described above cannot be mathematically inverted. But, the forward calculation and nonlinear fitting can be used to "invert" the problem. Given a set of measured and estimated parameters, a forward calculation is performed to generate a set of resonance frequencies, which are then compared to the measured values. Parameters are adjusted during each iteration to minimize the error function, which is defined as the sum of the squares of the differences between the calculated and measured frequencies.

The minimization of the error function is a nonlinear least-squares problem. The Levenberg-Marquart (LM) method is a classic algorithm for nonlinear least-squares problems and is typically employed in RUS analysis. The LM algorithm is used to find the set of adjustable parameters which minimizes the sum of squares of the differences between the calculated and measured frequencies

$$
F(x)=\sum_{i=1}^{M} w_{i}\left[f_{i}^{(\text {meas })}(x)-f_{i}^{(\text {calc })}(x)\right]^{2},
$$

where $f_{i}^{(\text {meas })}(x)$ are the measured frequencies, and $f_{i}^{(\text {calc })}(x)$ are the calculated ones. $w_{i}$ are the weights that reflect the confidence in the measured frequencies. These weights can be any value between 0 and 1 but they are usually all set to one except for the fundamental (lowest frequency) mode. The fundamental frequency consistently does not fit very well. The reason for this is not fully understood; however, there is evidence that it is due to an increased sensitivity to loading effects by the transducers [31]. In nonlinear fitting, appropriate starting values are important for the estimation of the model parameters. In addition to elastic constants, other parameters such as two out of the three dimensions (subject to a constant volume condition) or even Euler angles defining the crystal orientation relative to the sample geometry can be adjustable.

Inverse problems are typically ill-posed. Estimating elastic constants from the measured frequencies is no exception. A computer code was developed by Migliori et al. to handle the inverse problem in RUS [2]. One or more resonance modes may be missed during measurement if the pickup transducer touches the node of a mode, or if the transducer is insensitive to the direction a mode moves. If this occurs, one can identify the missing modes by comparing the measured and calculated frequencies after a few iterations of code. Errors for modes higher than the missing mode tend to be the same sign, either positive or negative. Inserting a missing mode or a place holder in the input file may quickly reduce the error. It is a good practice to start with the first few modes and add more mode(s) gradually. For a geometrically precise (errors in parallelism and perpendicularity of a few parts in $10^{3}$ ), high-Q (a few thousands or higher) sample, the overall RMS error between the calculated and measured frequencies is typically in the range of $0.1-0.3 \%$. Table 3 shows the first 20 modes in a typical "good fit" in RUS calculation for an $n$-type silicon-germanium ( $\mathrm{SiGe}$ ) sample near $500^{\circ} \mathrm{C}$.

\section{Experimental Methods}

3.1. Introduction. RUS involves the study of resonances of a properly prepared sample. A carefully polished sample, often a rectangular parallelepiped, is placed between a pair of acoustic transducers, one drives the sample in a sweptfrequency continuous wave $(\mathrm{CW})$ mode, the other detects the sample response. When the drive frequency matches a natural vibration frequency of the sample, the surface displacement amplitudes increase. The amount of increase depends on the quality factor $(Q)$ of the resonance, which is associated with the internal friction at that particular resonance frequency. RUS offers the advantages of point contact or loose coupling over the pulse-echo method, and can obtain all elastic constants in one measurement. 
TABle 3: Measured ( $\left.f^{\text {meas }}\right)$ and calculated $\left(f^{\text {calc }}\right)$ resonance frequencies after several iterations in a RUS calculation for an $n$-type silicon-germanium sample near $500^{\circ} \mathrm{C}$. The first mode was not included (given a weight of 0 ) in the final fit.

\begin{tabular}{lccc}
\hline Mode & $f^{\text {meas }}(\mathrm{MHz})$ & $f^{\text {calc }}(\mathrm{MHz})$ & Difference $(\%)$ \\
\hline 1 & 0.395133 & 0.393603 & 0.389 \\
2 & 0.540797 & 0.541167 & -0.068 \\
3 & 0.638239 & 0.638511 & -0.043 \\
4 & 0.642020 & 0.642575 & -0.086 \\
5 & 0.651254 & 0.651505 & -0.039 \\
6 & 0.709419 & 0.709383 & 0.005 \\
7 & 0.734763 & 0.735937 & -0.160 \\
8 & 0.739370 & 0.739131 & 0.032 \\
9 & 0.751041 & 0.749752 & 0.172 \\
10 & 0.758227 & 0.758263 & -0.005 \\
11 & 0.808084 & 0.807730 & 0.044 \\
12 & 0.835150 & 0.836441 & -0.154 \\
13 & 0.860419 & 0.861814 & -0.162 \\
14 & 0.880744 & 0.882067 & -0.150 \\
15 & 1.020977 & 1.020704 & 0.027 \\
16 & 1.030651 & 1.031488 & -0.081 \\
17 & 1.033333 & 1.034599 & -0.122 \\
18 & 1.070578 & 1.071760 & -0.110 \\
19 & 1.079524 & 1.077915 & 0.149 \\
20 & 1.094728 & 1.093729 & 0.091 \\
\hline
\end{tabular}

The sample peaks in the frequency domain are fit with a Lorentzian line shape to extract the center frequencies and quality factors. A complete vibration spectrum comprises the ordered list of these measured vibration frequencies of the sample. The measured spectrum is then compared to a spectrum computed from known parameters of dimensions, density, and initial values for elastic constants, which are adjusted in a least-squares sense to best fit the measured data. A block diagram of RUS is given in Figure 3.

The RUS code treats a variety of sample geometries but parallelepipeds remain as the most common ones. Raw materials of any geometry can be cut into parallelepiped shape using, low-speed diamond saw. The parallelepiped specimens are polished using a lapping machine. The detailed procedures for sample preparation can be found in the literature[31, 38, 39].

The piezoelectric effect of crystals and composite materials finds useful applications in the production and detection of sound. Although electromagnetic [40,41], laser optical [42-46], and other types of transducers [47] have been used for high-temperature RUS measurements, piezoelectric materials are good choices due to their advantages such as efficiency, simplicity, and low design cost.

In a RUS measurement, the absolute amplitude of a resonance depends on many factors such as the amplitude of driving signal and the loading (contact force between the sample and the transducers) to the sample. Although the amplitude information is unreliable and not used in RUS calculation, it is desired that transducers have a wide, flat frequency response in the frequency range of operation. An ultrasonic transducer for RUS measurement differs fundamentally from conventional ultrasonic transducers in which resonances of the transducer itself should be small compared to sample resonances, and if possible, should be kept out of frequency range of operation [48, 49]. Various transduction systems have been used to accommodate samples of various types and sizes in RUS measurement. We present readers a few samples.

Novel and exotic materials in crystal form usually come in small quantities and sizes, and are often quite expensive to synthesize. RUS excels at handling sub-mm-sized small samples. In an ideal RUS measurement, the drive transducer and pickup transducer are in contact with the two diagonally opposite corners of a parallelepiped sample so all resonance modes can be detected. The sharp corners of a mmsized sample can be easily damaged if held by transducers with hard faces. PVDF film is a flexible, lightweight, and tough plastic material that can be electrically polarized for piezoelectric applications. PVDF film transducers offer advantages of wide frequency and dynamic range over other traditional ceramic transducers, and are particularly suited for handling small crystals with sharp corners. Maynard designed a small transducer cell using PVDF film transducers [28]. When a parallelepiped sample is mounted in this transducer cell, the two diagonally opposite corners of the sample are held between the two flexible transducers. A small transducer cell similar to Maynard's cell has been constructed at the National Center for Physical Acoustics (NCPA). The photograph of the cell is shown in Figure 4. The tension between the sample and transducers can be finely adjusted by advancing the sliding block to adjust the separation between the transducer strips. Due to the close distance between the two parallel PVDF transducers, electrical crosstalk can be troublesome when sample resonances are weak. A piece of grounded copper shield, with a center hole slightly larger than the sample, is placed between the two transducers. This small sample RUS apparatus is also useful for making measurements of heterogeneous samples composed of a substrate and thin film $[31,32,50]$.

\subsection{High-Temperature Resonant Ultrasound Spectroscopy.} RUS is commonly used for characterizing elastic properties of a wide variety of materials at room and low temperatures. Applications at elevated temperatures, however, are relatively rare [51] and have mostly been concentrated in the geophysics community. Some added technical difficulties in high-temperature measurements are (i) sensitivity of most piezoelectric transducers decreases, if not rapidly, with increasing temperature, thus making them unusable above certain temperatures, (ii) strength of the acoustic resonance of most materials becomes weak at high temperatures, and the broadened sample resonances due to increased attenuation and internal friction in materials can make it difficult to separate sample modes; (iii) technical challenges posed by electrical connections, transducer housing, and other related issues need to be considered. 


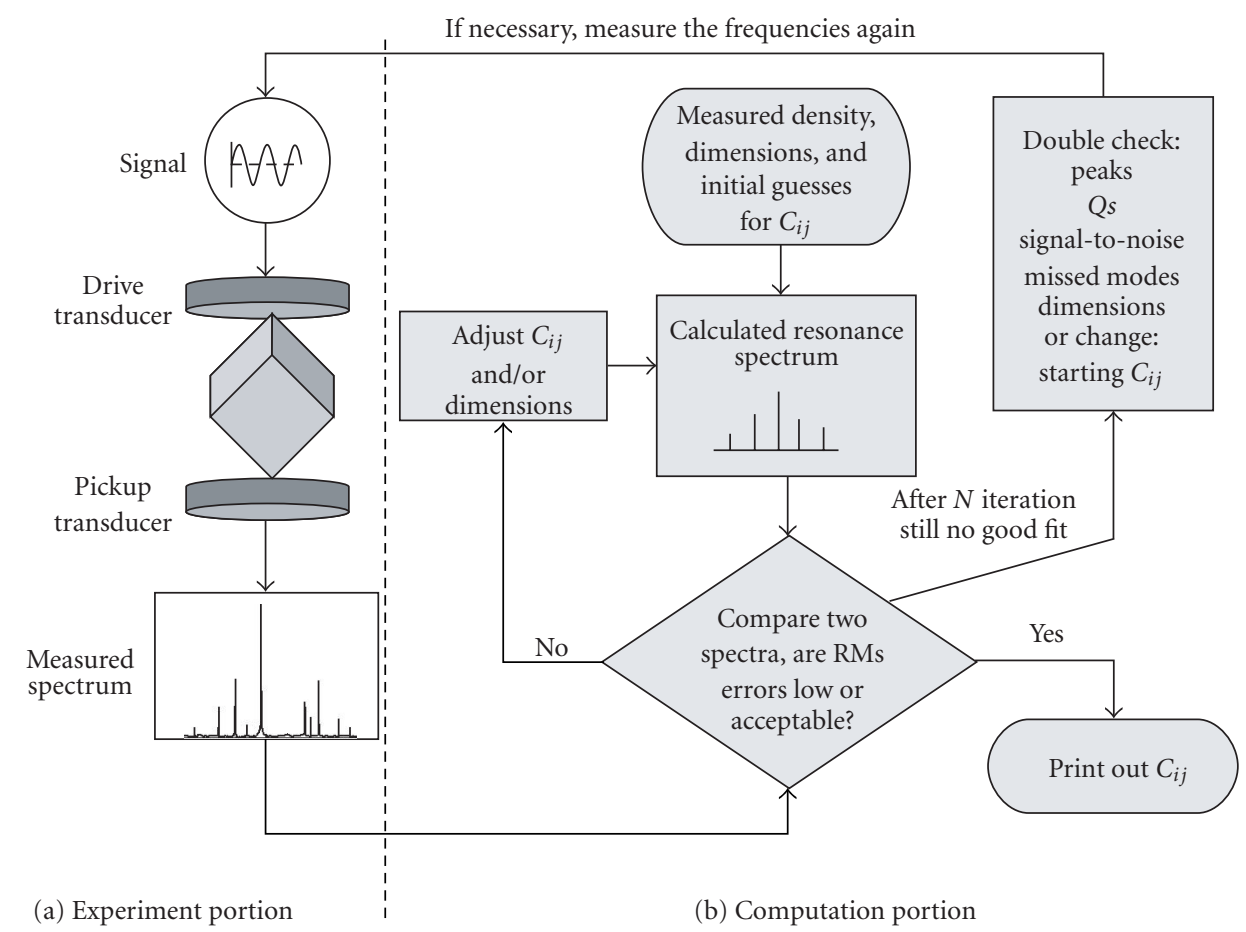

FIGURE 3: Schematic block diagram of RUS measurement and computation. (a) represents the experiment portion: prepare the sample, excite it, and detect sample resonances. (b) represents the computation portion: extract center frequencies and Qs, compute resonance spectrum from estimated parameters then compare it to the measured spectrum, adjust the parameters, and iterate toward a good fit to the measured spectrum so elastic constants are obtained.

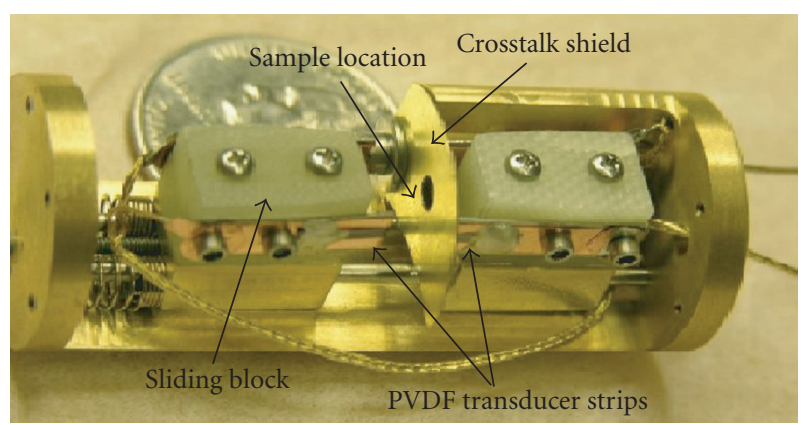

Figure 4: Photograph of the small RUS cell using two PVDF transducers. See the text for a description of the components.

Despite early successes of RUS applications in extreme environments such as high temperatures $[15-17,19,51-$ 53], recent applications reported in the literature are scarce. Refractory materials such as corundum $\left(\mathrm{Al}_{2} \mathrm{O}_{3}\right)$ and periclase $(\mathrm{MgO})$ can be placed in an open furnace for hightemperature studies. For oxidation-sensitive materials, a sealed system with minimum trace oxygen is required. In order to study high-temperature materials of weak resonances, a specialty transducer system was also developed at NCPA. The system has been used successfully up to $600^{\circ} \mathrm{C}$. For higher-temperature measurements, a buffer-rod system was used at temperatures up to $1000^{\circ} \mathrm{C}$ of the furnace temperature limit.
3.2.1. Inert Gas Flushing System. In high-temperature RUS measurement of oxidation-sensitive materials, a full temperature run can take dozens of hours. To minimize sample exposure to oxygen at high temperatures, an inert gas flushing system is employed at NCPA to maintain a low-oxygen environment during measurement. This system consists of (a) a compressed inert gas source (typically argon), (b) a gas regulator, (c) an oxygen trap, (d) an oxygen trace analyzer, and (e) a copper cooling coil. During measurement, the sample and transducer cell are placed inside the quartz tube, which snuggly runs through the high-temperature tube furnace, and gas flow is maintained at about 1 liter per minute. The schematic diagram of the gas flushing system is given in Figure 5.

\subsubsection{Transducer Systems for High-Temperature RUS Mea-} surement. The upper operating temperature of a piezoelectric transducer can be limited by several factors: (i) most materials that exhibit good piezoelectric properties at room or low temperatures lose the piezoelectric effect quickly with increasing temperature; (ii) the operating temperatures in crystals with high Curie temperatures are usually well below their Curie temperatures due to impurities in their crystalline structures; (iii) the upper temperature limit may also be restricted by transducer components such as backing and supporting materials, bonding materials, and electrical connections. 
(e)
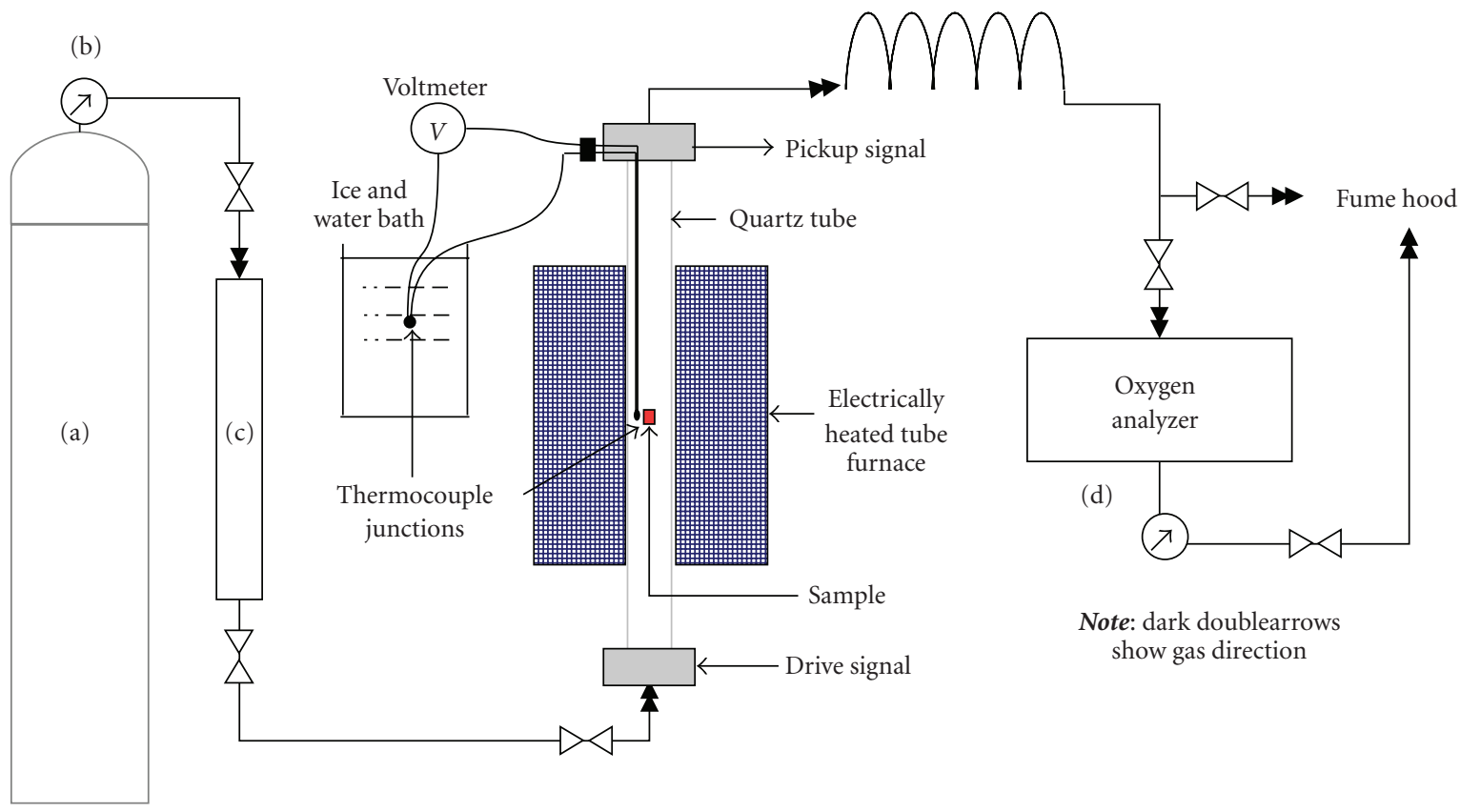

Note: dark doublearrows show gas direction

Figure 5: Schematic diagram of the inert gas flushing system. The whole system consists of (a) a compressed inert gas source, (b) a gas regulator, (c) an oxygen trap, (d) an oxygen trace analyzer, (e) a copper cooling coil, and miscellaneous items such as a purge valve and other valves.

Most common piezoelectric materials such as lead zirconate titanate (PZT) ceramics are not suited for hightemperature applications. Many piezoelectric materials have been investigated for scientific and industrial uses at hightemperatures [54-56]. Several issues need to be considered when applying a piezoelectric material for high temperature applications [54]: the operating temperature of a material is often well below its Curie transition temperature, at which the material loses piezoelectric property; many piezoelectric materials are also pyroelectric, temperature variations may induce charges; dramatically increased electrical conductivity may result in charge drift and partial loss of signal, the conductivity problem is often aggravated in low-oxygen environment. Potential candidates for high-temperature $\left(600^{\circ} \mathrm{C}\right.$ and above) piezoelectric applications include tourmaline, bismuth titanate $\left(\mathrm{Bi}_{4} \mathrm{Ti}_{3} \mathrm{O}_{12}\right)$, gallium orthophosphate $\left(\mathrm{GaPO}_{4}\right)$, aluminum nitride (AlN), lithium niobate $\left(\mathrm{LiNbO}_{3}\right)$, lanthanum gallium silicate (langasite, LGS), and lanthanum gallium tantalate (langatate, LGT) [57]. The high Curie temperature of $\mathrm{LiNbO}_{3}$, above $1200^{\circ} \mathrm{C}$, along with its wide availability from commercial suppliers, makes it a good candidate for high-temperature RUS applications [58]. Pure $\mathrm{LiNbO}_{3}$ crystals with stoichiometric compositions are reported to exhibit remarkable piezoelectric properties even at $900^{\circ} \mathrm{C}[59,60]$; however, typical upper temperature limits for lithium niobate transducers are about $550^{\circ} \mathrm{C}$ (see below). Single $y / 36^{\circ}$-cut crystals with chrome/gold deposited on both sides were chosen as the piezoelectric elements for vibration excitation and detection. The mineral-insulated (MI) signal transmission cables made by ThermoCoax provide electrical connections to the piezoelectric elements. These coax- ial cables are designed for high-temperature environment (above $1000^{\circ} \mathrm{C}$ ). A silver-filled conductive epoxy (Epo-Tek E2101) is a good conductor at high temperatures and can provide good bonds between the electrode of piezoelectric element and MI cable.

The schematic of this direct-contact transducer probe can be found in Figure 6. The direct-contact scheme has advantages over the buffer-rod system: much improved signal-to-noise ratio (SNR); clean sample resonance signals without additional problematic resonances from the buffer rods. Unfortunately, the piezoelectric lithium niobate loses sensitivity beginning $\sim 600^{\circ} \mathrm{C}$. The lithium niobate acoustic transducers were reported be able to operate at temperatures close to the Curie temperature $[16,17]$. However, there is no further reported use of $\mathrm{LiNbO}_{3}$ for similar resonance measurement at such high temperatures. Many recent evidences in the literature, however, show that the typical upper operating temperature of $\mathrm{LiNbO}_{3}$ is about $650^{\circ} \mathrm{C}$, which is far below the material's theoretical Curie temperature of above $1200^{\circ} \mathrm{C}[56,58]$. It has been discussed in the literature [56] that this signal deterioration is caused by oxygen loss, and it might have been exacerbated in lowoxygen environment. Other factors such as impurities and crystal stoichiometry may also have some effect $[59,60]$. The presence of defects in the crystal lattice can significantly affect the material's piezoelectric properties at high temperatures. The silver-filled epoxy can provide electrical connection and mechanical bond up to $800-900^{\circ} \mathrm{C}$ range. With new piezoelectric materials of higher operating temperatures, upper operating temperature of a transducer probe with similar design can be pushed to $900^{\circ} \mathrm{C}$. 


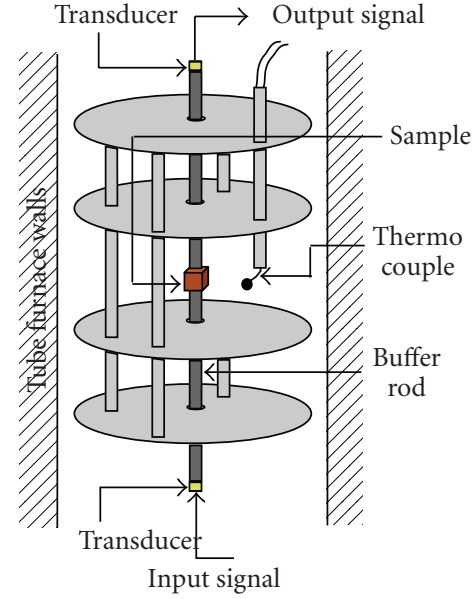

(a)

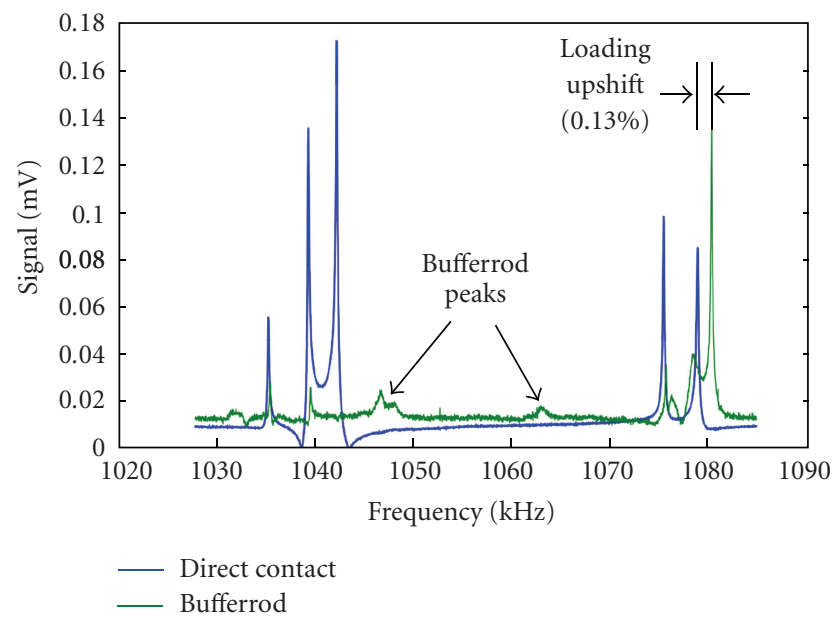

(c)

FIGURE 6: (Color online) Schematic of (a) buffer rod and (b) direct contact systems and (bottom) typical resonance data acquired for each system. The sample in this case was SiGe and both spectra were obtained at $410^{\circ} \mathrm{C}$.

The current restrictions by the piezoelectric elements in the direct-contact transducer system can be overcome using buffer-rod systems [61, 62]. Acoustic buffer rods have long been used for making high-temperature ultrasonic measurement including elasticity measurements [63, 64]. The conventional buffer-rod system makes RUS measurement possible at extremely high temperatures, and such applications have been reported in the literature $[19,51,52]$. The buffer rods, usually made out of materials of low acoustic loss such as metals, ceramics, or fused quartz, serve as waveguides for ultrasound transmission.

A buffer-rod transducer system was constructed at NCPA for high-temperature RUS measurement. In the buffer-rod probe, the piezoelectric element is cemented to one end of a $0.318 \mathrm{~cm}$ diameter buffer rod; the other end of the buffer rod is in direct contact with the test sample placed in the hightemperature environment. In operation, the transducer is exposed to a temperature that is significantly lower than that of the sample. A pair of buffer-rod probes are needed for RUS measurement: one to drive the sample, the other to detect the sample resonance. A cage consists of alumina ceramic disks and rods provides structural support for the two buffer-rod probes and the thermocouple wire. The disks and rods are cemented together using high-temperature chemical set cement (Omegabond OB-700). The cage can withstand repeated high temperatures and retain rigidity in the whole operating temperature range up to $1000^{\circ} \mathrm{C}$. The resonances of the cage are weak, and this feature is highly desired in RUS measurement. When the whole apparatus is loaded into the tube furnace, flexible high-temperature (rated up to $200^{\circ} \mathrm{C}$ ) coaxial cables are used to connect the piezoelectric elements to coaxial feedthroughs. The flexible cable allows the upper transducer probe to move up and down freely in the cage so no extra force in addition to the weight of the upper transducer probe is exerted on the test sample. The schematic for the buffer-rod system is shown in Figure 6.

The added temperature range afforded by the buffer rod system comes at a cost, however. The acoustic signal transmitted through the long buffer rod loses some strength, and this is particularly detrimental to the measurement of weak resonance signals. Radial resonances of buffer rods can be excited and mixed in with sample peaks, making the identification of sample peaks difficult and time consuming. Lastly, the extra weight of the buffer rod $(\sim 7 \mathrm{~g})$ causes a slight upshifting of resonances which is mode dependent, but typically not more than $0.1 \%$. The spectra shown in Figure 6 illustrates these issues.

\section{Applications}

4.1. Elastic Constant Measurement of Small and LowSymmetry Crystals. As a versatile tool for charactering elastic properties of solid materials, RUS has found applications in a variety of fields. Materials scientists are interested in mechanical properties of their newly developed and modified materials that are often available in small quantities and sizes. RUS measures $\mathrm{mm}$-sized samples handily $[28,31]$. The use of a small sample of a material is more likely to guarantee homogeneity. Thin film materials have been the subject of intense research in recent years, and RUS analysis has been extended to handle heterogeneous samples such as a thin film deposited on a substrate. The knowledge of elastic properties of thin film materials are required for a variety of applications. RUS has been used to study colossal magnetoresistance (CMR) and silicon carbide thin (200$1600 \mathrm{~nm}$ ) films deposited on a substrate $[31,50]$.

\subsection{Elastic Constant Measurements at High Temperatures.} Knowledge of the mechanical properties of materials intended for use in high-temperature environments is required for understanding the fundamental physics of these materials [65] and is crucial information for proper mechanical design. Applications of resonance techniques at elevated temperatures have been reported in the literature [15-17, 19, 51-53, 66]. Early high-temperature RUS applications were mostly limited to the geophysical studies of 
the Earth. Geophysicists are particularly interested in the elastic behaviors of constituent phases in Earth's mantle at temperatures beyond their Debye temperatures [67]. The high-temperature data of geophysically important minerals is needed for studying the thermal equation of state, the structure, and the composition in the Earth's interior.

Two main transducer systems were used in these hightemperature studies of geophysical materials. An apparatus with piezoelectric transducers was used by Sumino et al. to evaluate the temperature variations of elastic constants of single-crystal forsterite $\left(\mathrm{Mg}_{2} \mathrm{SiO}_{4}\right)$ up to about $400^{\circ} \mathrm{C}$ [15]. In this apparatus, the sample is in direct contact with the two acoustic transducers, and the load on the sample is kept at minimum using a microbalance. The transducer and sample assembly in a vacuum chamber are heated by an electric furnace. A similar apparatus was reported to obtain the elastic constants of a single crystal forsterite $\left(\mathrm{Mg}_{2} \mathrm{SiO}_{4}\right)$ from room temperature to about $927^{\circ} \mathrm{C}$ [17], and the elastic constants of a single crystal periclase $(\mathrm{MgO})$ up to $1027^{\circ} \mathrm{C}[16]$. In the direct-contact setup, both the sample and transducers experience the same high temperature, so the upper operating temperature is often limited by the piezoelectric elements. For higher-temperature measurement, the transducers need to be placed outside of the extremely hightemperature environment. A buffer-rod RUS apparatus was used for measuring elastic constants of single crystals up to $1552^{\circ} \mathrm{C}$ in this system [19]. The single crystal corundum $\left(\mathrm{Al}_{2} \mathrm{O}_{3}\right)$ parallelepiped was held between two long, thin alumina buffer rods, which separate the $\mathrm{BaTiO}_{3}$ transducers attached at the other ends of buffer rods from the harsh high-temperature environment. The load of the upper buffer rod on the sample was fine adjusted using a beam balance. The buffer-rod system was also used to obtain the elastic constants of single-crystal forsterite $\left(\mathrm{Mg}_{2} \mathrm{SiO}_{4}\right)$ up to $1427^{\circ} \mathrm{C}$ [21] and periclase $(\mathrm{MgO})$ up to $1527^{\circ} \mathrm{C}$ [20].

4.3. Temperature-Induced Phase Transition Studies. Elastic constants are a measure of the curvature of the bonding energy within a crystal structure near the minimum and are sensitive probes to any changes in the atomic environment. Subtle changes often missed by other experimental methods can be observed in RUS measurements. Such phase transitions can come in many forms-structural, superconducting, magnetic, charge ordering, glass, and so forth. The measurement of elasticity of a solid as a function of temperature has been a traditional and useful technique for studying temperature-induced phase transitions. The exact nature of the temperature trends of elastic constants near the critical temperature depends on the specifics of the transition. However, they generally are exhibited as either a discontinuous shift in the values of the elastic constants themselves or in the derivative with respect to temperature. Frequency measurements can be as precise as several parts per million in a RUS experiment, making it an extremely precise method of determining elastic constants of crystalline and noncrystalline materials. The high precision makes RUS a sensitive and powerful tool for studying various phase transitions $[2,22,23,68]$. RUS is complementary to other conventional techniques such as X-ray and neutron techniques for phase transition studies.

Migliori et al. successfully studied the elastic behavior of $\mathrm{La}_{1.86} \mathrm{Sr}_{0.14} \mathrm{CuO}_{4}$ near superconducting transition temperature (about $220 \mathrm{~K}$ ) [23]. A giant softening in $C_{66}$ accompanied the tetragonal-orthorhombic (TO) structural phase transition (SPT). In addition, they also applied RUS to SPT studies in $\mathrm{SrTiO}_{3}$ and $\mathrm{La}_{2} \mathrm{CuO}_{4}$ [2]. McGuire et al. experimentally investigated phase transitions in superconductor LaFeAsO using various techniques including RUS [69]. An obvious gradual softening in the elastic moduli was observed, and the transition temperature was determined using the temperature derivative of the elastic moduli. Ohno et al. studied the well-known $\alpha-\beta$ transition in quartz near $573^{\circ} \mathrm{C}$ using a buffer-rod system [53]. The complete set of elastic constants of $\alpha$ - and $\beta$-quartz were determined as function of temperature, and the softening of elastic constants near the transition temperature was clearly observed.

4.4. Nanostructured Doped Silicon Germanium. Our group has recently concluded a study of doped nanostructured silicon germanium ( $\mathrm{SiGe}$ ) which has potential applications as a high-temperature thermoelectric material [70]. We measured the elastic moduli of both phosphorous doped ( $n$ type) and boron-doped ( $P$-type) silicon germanium which was fabricated using a hot press technique at the Jet Propulsion Laboratory. The resulting material was polycrystalline with crystallite sizes of about $70 \mathrm{~nm}$, much smaller than an acoustic wavelength $(\sim 1.0 \mathrm{~mm})$, so that an isotropic elastic model could accurately be used. The final sample compositions were $\mathrm{Si}_{0.784} \mathrm{Ge}_{0.196} \mathrm{P}_{0.020}$ for the $n$-type samples and $\mathrm{Si}_{0.787} \mathrm{Ge}_{0.197} \mathrm{~B}_{0.016}$ for the $p$-type samples. We found a rather dramatic difference in the temperature dependence of the moduli between the $n$ - and $p$-type SiGe which is shown in Figure 7 . The $p$-type samples show a smooth softening with a slight negative curvature over the entire temperature range with no hysteresis observed upon heating/cooling cycles. The $n$-type sample, however, shows a rather sharp stiffening near $650 \mathrm{~K}$ followed by a more pronounced softening beginning at about $800 \mathrm{~K}$ during the initial heating. Upon cooling, the moduli smoothly stiffen and ultimately end slightly stiffer after the thermal cycle is complete. If the sample is reheated, the moduli follow the previous cooling trend, the stiffening feature is not repeated.

We attributed the stiffening in the $n$-type sample to precipitation of the phosphorous atoms in Si-Ge matrix. Phosphorous in silicon germanium exhibits a retrograde solubility limit in the range of $600-1273 \mathrm{~K}$ [71]. As the phosphorous atoms precipitate out of the Si-Ge, the ratio of $\mathrm{Si} / \mathrm{Ge}-\mathrm{P}$ to $\mathrm{Si} / \mathrm{Ge}-\mathrm{Si} / \mathrm{Ge}$ bonds drops as more $\mathrm{P}$ atoms are bonded to other $\mathrm{P}$ atoms rather than the host matrix, and the $\mathrm{Si}-\mathrm{Ge}$ to $\mathrm{Si} / \mathrm{Ge}$ potentials have a sharper curvature than the $\mathrm{Si} / \mathrm{Ge}-\mathrm{P}$ potentials resulting in an overall stiffer material. The precipitation is not reversible in the sense that when the material cools, much of the phosphorous remains precipitated out, and the material is stiffer at room temperature after thermal cycling. This hypothesis was tested by heating the samples to $1275 \mathrm{~K}$ for 30 minutes and then air 


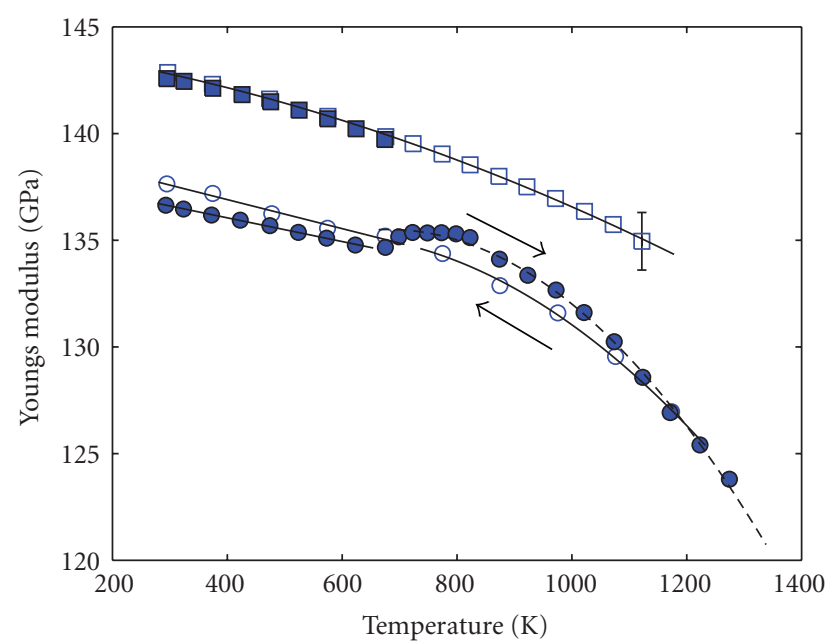

(a)

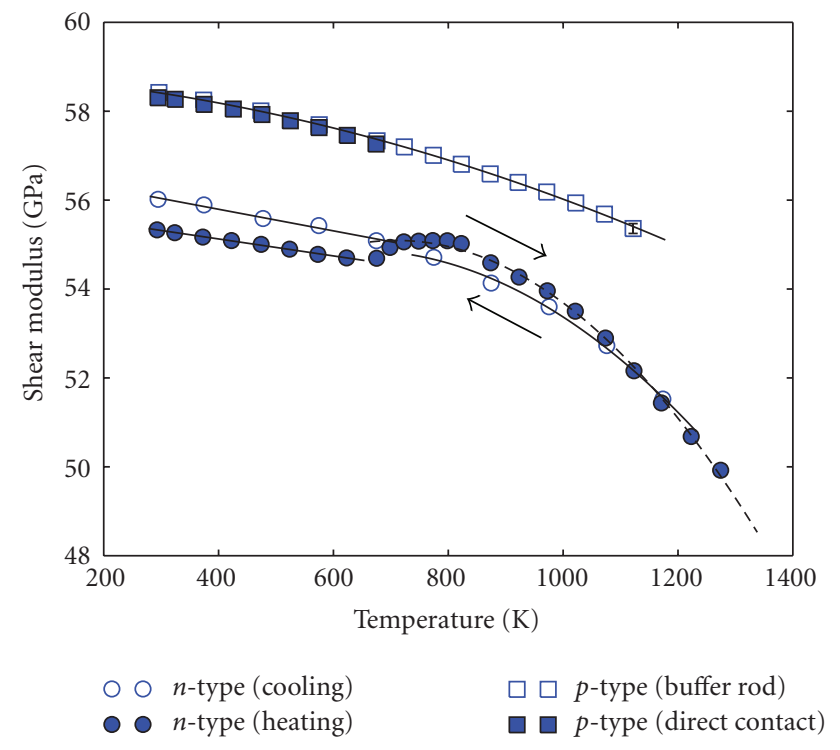

(b)

Figure 7: Isotropic elastic moduli of $n$ - and $p$-type from room temperature to about $1250 \mathrm{~K}$ (reprinted from [70]).

quenching them to room temperature. At this temperature, the solubility is maximum, and the phosphorous redissolves into the $\mathrm{Si} / \mathrm{Ge}$ matrix. When this heat-treated sample was remeasured using RUS, the temperature dependence of the moduli was precisely repeated-the stiffening was observed on heating and was not observed on cooling. Further evidence for our conclusion comes from electrical resistivity and Seebeck coefficient measurements which show similar anomalies at $650 \mathrm{~K}$ and hysteresis in the $n$-type samples but not the $p$-type. This particular application of RUS exemplifies the wealth of information which can be derived from elastic constant measurements. The original goal of the project was simply to characterize the thermomechanical properties of these materials to help design thermoelectric devices and understand thermal stresses imposed by the temperature gradients. While that goal was achieved, more fundamental physics was also learned.

\section{Conclusions}

We have presented here a review of the experimental method of resonant ultrasound spectroscopy to measure the elastic tensor of a material. We have focused on high-temperature methods which are becoming increasingly important as new energy related materials with operating environments well above room temperature are being actively researched. Since its development as an experimental technique in the early 1990's, RUS has become the preferred method for elastic constant measurements, and the community of practitioners is expanding.

\section{Acknowledgment}

J. R. Gladden acknowledges the Department of Energy, EPSCoR Award no. DE-FG02-04ER46121, NASA Award no. NM0710850, and the University of Mississippi for support of this project as well as P. Smith for fruitful discussions.

\section{References}

[1] R. F. S. Hearmon, "The elastic constants of anisotropic materials," Reviews of Modern Physics, vol. 18, no. 3, pp. 409440, 1946.

[2] A. Migliori, J. L. Sarrao, W. M. Visscher et al., "Resonant ultrasound spectroscopic techniques for measurement of the elastic moduli of solids," Physica B, vol. 183, no. 1-2, pp. 1-24, 1993.

[3] H. Czichos, Springer Handbook of Materials Measurement Methods, Edited by H. Czichos, T. Saito, L. Smith, Springer, Berlin, Germany, 2006.

[4] R. Truell, C. Elbaum, and B. B. Chick, Ultrasonic Methods in Solid State Physics, Academic Press, New York, NY, USA, 1969.

[5] M. F. Markham, "Measurement of elastic constants by the ultrasonic pulse method," British Journal of Applied Physics, vol. 8, no. 6, article 312, pp. S56-S63, 1957.

[6] J. W. S. Rayleigh, The Theory of Sound, MacMillan, London, UK, 2nd edition, 1894.

[7] R. D. Mindlin, "Simple modes of vibration of crystals," Journal of Applied Physics, vol. 27, no. 12, pp. 1462-1466, 1956.

[8] D. B. Fraser and R. C. Lecraw, "Novel method of measuring elastic and anelastic properties of solids," Review of Scientific Instruments, vol. 35, no. 9, pp. 1113-1115, 1964.

[9] H. Ekstein and T. Schiffman, "Free vibrations of isotropic cubes and nearly cubic parallelepipeds," Journal of Applied Physics, vol. 27, no. 4, pp. 405-412, 1956.

[10] R. Holland, "Resonant properties of piezoelectric ceramic rectangular parallelepipeds," The Journal of the Acoustical Society of America, vol. 43, no. 5, p. 988, 1968.

[11] P. M. Morse and H. Feshbach, Methods of Theoretical Physics, McGraw-Hill, New York, NY, USA, 1953.

[12] H. H. Demarest Jr., "Cube-resonance method to determine the elastic constants of solids," Journal of the Acoustical Society of America, vol. 49, no. 3, pp. 768-775, 1971.

[13] I. Ohno, "Free vibration of a rectangular parallelepiped crystal and its application to determination of elastic constants of orthotropic crystal," Journal of Physics of the Earth, vol. 24, pp. 355-379, 1976. 
[14] Y. Sumino, I. Ohno, T. Goto, and M. Kumazawa, "Measurement of elastic constants and internal friction of single crystal MgO by rectangular parallelepiped resonance," Journal of Physics of the Earth, vol. 24, pp. 263-273, 1976.

[15] Y. Sumino, O. Nishizawa, T. Goto, I. Ohno, and M. Ozima, "Temperature variation of elastic constants of single crystal forsterite between- $190^{\circ}$ and $400^{\circ} \mathrm{C}$," Journal of Physics of the Earth, vol. 25, pp. 377-392, 1977.

[16] Y. Sumino, O. L. Anderson, and I. Suzuki, "Temperature coefficients of elastic constants of single crystal MgO between 80 and 1,300 K," Physics and Chemistry of Minerals, vol. 9, no. 1, pp. 38-47, 1983.

[17] I. Suzuki, O. L. Anderson, and Y. Sumino, "Elastic properties of a single-crystal forsterite $\mathrm{Mg}_{2} \mathrm{SiO}_{4}$, up to 1,200 K," Physics and Chemistry of Minerals, vol. 10, no. 1, pp. 38-46, 1983.

[18] I. Ohno, S. Yamamoto, O. L. Anderson, and J. Noda, "Determination of elastic constants of trigonal crystals by the rectangular parallelepiped resonance method," Journal of Physics and Chemistry of Solids, vol. 47, no. 12, pp. 1103-1108, 1986.

[19] T. Goto and O. L. Anderson, "Apparatus for measuring elastic constants of single crystals by a resonance technique up to 1825 K," Review of Scientific Instruments, vol. 59, no. 8, pp. 1405-1408, 1988.

[20] D. G. Isaak, O. L. Anderson, and T. Goto, "Measured elastic moduli of single-crystal $\mathrm{MgO}$ up to $1800 \mathrm{~K}$," Physics and Chemistry of Minerals, vol. 16, no. 7, pp. 704-713, 1989.

[21] D. G. Isaak, O. L. Anderson, T. Goto, and I. Suzuki, "Elasticity of single-crystal forsterite measured to $1700 \mathrm{~K}$," Journal of Geophysical Research, vol. 94, no. 5, pp. 5895-5906, 1989.

[22] A. Migliori, W. M. Visscher, S. E. Brown et al., "Elastic constants and specific-heat measurements on single crystals of $\mathrm{La}_{2} \mathrm{CuO}_{4}$," Physical Review B, vol. 41, no. 4, pp. 2098-2102, 1990.

[23] A. Migliori, W. M. Visscher, S. Wong et al., "Complete elastic constants and giant softening of c66 in superconducting $\mathrm{La}_{1.86} \mathrm{Sr}_{0.14} \mathrm{CuO}_{4}$," Physical Review Letters, vol. 64, no. 20, pp. 2458-2461, 1990.

[24] R. B. Schwarz and J. F. Vuorinen, "Resonant ultrasound spectroscopy: applications, current status and limitations," Journal of Alloys and Compounds, vol. 310, no. 1-2, pp. 243 250, 2000.

[25] B. J. Zadler, J. H. L. Le Rousseau, J. A. Scales, and M. L. Smith, "Resonant ultrasound spectroscopy: theory and application," Geophysical Journal International, vol. 156, no. 1, pp. 154-169, 2004.

[26] A. Migliori and J. D. Maynard, "Implementation of a modern resonant ultrasound spectroscopy system for the measurement of the elastic moduli of small solid specimens," Review of Scientific Instruments, vol. 76, no. 12, Article ID 121301, pp. $1-7,2005$.

[27] W. M. Visscher, A. Migliori, T. M. Bell, and R. A. Reinert, “On the normal modes of free vibration of inhomogeneous and anisotropic elastic objects," Journal of the Acoustical Society of America, vol. 90, no. 4, pp. 2154-2162, 1991.

[28] J. D. Maynard, "The use of piezoelectric film and ultrasound resonance to determine the complete elastic tensor in one measurement," Journal of the Acoustical Society of America, vol. 91, no. 3, pp. 1754-1762, 1992.

[29] D. G. Isaak and I. Ohno, "Elastic constants of chromediopside: application of resonant ultrasound spectroscopy to monoclinic single-crystals," Physics and Chemistry of Minerals, vol. 30, no. 7, pp. 430-439, 2003.
[30] H. Ledbetter, H. Ogi, and N. Nakamura, "Elastic, anelastic, piezoelectric coefficients of monocrystal lithium niobate," Mechanics of Materials, vol. 36, no. 10, pp. 941-947, 2004.

[31] J. R. Gladden, Characterization of thin films and novel materials by resonant ultrasound spectroscopy, Ph.D. thesis, The Pennsylvania State University, University Park, Pa, USA, 2003.

[32] J. H. So, J. R. Gladden, Y. H. Hu, J. D. Maynard, and Q. Li, "Measurements of elastic constants in thin films of colossal magnetoresistance material," Physical Review Letters, vol. 90, no. 3, Article ID 036103, pp. 036103/1-036103/4, 2003.

[33] A. E. H. Love, A Treatise on the Mathematical Theory of Elasticity, Cambridge University Press, Cambridge, Mass, USA, 3rd edition, 1920.

[34] I. S. Sokolnikoff, Mathematical Theory of Elasticity, McGrawHill, New York, NY, USA, 2nd edition, 1956.

[35] L. D. Landau and E. M. Lifshitz, Theory of Elasticity, AddisonWesley, Boston, Mass, USA, 1959, Translated from the Russian by J. B. Sykes and W. H. Reid.

[36] S. P. Timoshenko and J. N. Goodier, Theory of Elasticity, McGraw Hill, New York, NY, USA, 3rd edition, 1970.

[37] J. Plesek, R. Kolman, and M. Landa, "Using finite element method for the determination of elastic moduli by resonant ultrasound spectroscopy," Journal of the Acoustical Society of America, vol. 116, no. 1, pp. 282-287, 2004.

[38] P. S. Spoor, Elastic properties of novel materials using PVDF film and resonance ultrasound spectroscopy, Ph.D. thesis, The Pennsylvania State University, 1997.

[39] A. Migliori and J. L. Sarrao, Resonant Ultrasound Spectroscopy: Applications to Physics, Materials Measurements, and Nondestructive Evaluation, Wiley-Interscience, Hoboken, NJ, USA, 1997.

[40] M. Hirao and H. Ogi, "Electromagnetic acoustic resonance and materials characterization," Ultrasonics, vol. 35, no. 6, pp. 413-421, 1997.

[41] H. Ogi, H. Ledbetter, S. Kim, and M. Hirao, "Contactless mode-selective resonance ultrasound spectroscopy: electromagnetic acoustic resonance," Journal of the Acoustical Society of America, vol. 106, no. 2, pp. 660-665, 1999.

[42] C. B. Scruby and L. E. Drain, Laser Ultrasonics: Techniques and Applications, Taylor \& Francis, London, UK, 1990.

[43] S. Sato, K. Inagaki, V. E. Gusev, and O. B. Wright, "Resonant ultrasound spectroscopy using optical excitation and detection," in Proceedings of the 10th International Conference on Photoacoustic and Photothermal Phenomena, vol. 463 of AIP Conference Proceedings, pp. 424-426, 1999.

[44] H. Ogi, Y. Kawasaki, M. Hirao, and H. Ledbetter, "Acoustic spectroscopy of lithium niobate: elastic and piezoelectric coefficients," Journal of Applied Physics, vol. 92, no. 5, p. 2451, 2002.

[45] H. Ogi, K. Sato, T. Asada, and M. Hirao, "Complete mode identification for resonance ultrasound spectroscopy," Journal of the Acoustical Society of America, vol. 112, no. 6, pp. 25532557, 2002.

[46] P. Sedlák, M. Landa, H. Seiner, L. Bicanová, and L. Heller, "Non-contact resonant ultrasound spectroscopy for elastic constants measurement," in Proceedings of 1st International Symposium on Laser Ultrasonics: Science, Technology and Applications (LU'08), Montréal, Canada, 2008.

[47] V. T. Kuokkala and R. B. Schwarz, "The use of magnetostrictive film transducers in the measurement of elastic moduli and ultrasonic attenuation of solids," Review of Scientific Instruments, vol. 63, no. 5, pp. 3136-3142, 1992. 
[48] A. Migliori and T. W. Darling, "Resonant ultrasound spectroscopy for materials studies and non-destructive testing," Ultrasonics, vol. 34, no. 2-5, pp. 473-476, 1996.

[49] H. Mrasek, D. Gohlke, K. Matthies, and E. Neumann, "Ultrasound transducers for 650 centigrade continuous application," Materials Testing, vol. 38, no. 10, pp. 416-418, 1996.

[50] K. A. Pestka II, J. D. Maynard, D. Gao, and C. Carraro, "Measurement of the elastic constants of a columnar $\mathrm{SiC}$ thin film," Physical Review Letters, vol. 100, no. 5, Article ID 055503, 2008.

[51] O. L. Anderson, "Rectangular parallelepiped resonance-a technique of resonance ultrasound and its applications to the determination of elasticity at high temperatures," Journal of the Acoustical Society of America, vol. 91, no. 4, pp. 2245-2253, 1992.

[52] T. Goto, O. L. Anderson, I. Ohno, and S. Yamamoto, "Elastic constants of corundum up to $1825 \mathrm{~K}$," Journal of Geophysical Research, vol. 94, no. 6, pp. 7588-7602, 1989.

[53] I. Ohno, K. Harada, and C. Yoshitomi, "Temperature variation of elastic constants of quartz across the $\alpha-\beta$ transition," Physics and Chemistry of Minerals, vol. 33, no. 1, pp. 1-9, 2006.

[54] R. C. Turner, P. A. Fuierer, R. E. Newnham, and T. R. Shrout, "Materials for high temperature acoustic and vibration sensors: a review," Applied Acoustics, vol. 41, no. 4, pp. 299-324, 1994.

[55] V. Giurgiutiu, X. Buli, C. Jaycee, and J. Laskis, “Development of a wireless active system for TPS structural health monitoring," in Quantitative Nondestructive Evaluation, vol. 820 of AIP Conference Proceedings, pp. 1764-1771, 2006.

[56] R. Kažys, A. Voleišis, and B. Voleišienè, "High temperature ultrasonic transducers: review," Ultragarsas (Ultrasound), vol. 63, no. 2, pp. 7-17, 2008.

[57] E. Ansorge, S. Schimpf, S. Hirsch, J. Sauerwald, H. Fritze, and B. Schmidt, "Evaluation of langasite $\left(\mathrm{La}_{3} \mathrm{Ga}_{5} \mathrm{SiO}_{14}\right)$ as a material for high temperature microsystems," Sensors and Actuators, A, vol. 130-131, pp. 393-396, 2006.

[58] N. Schmarje, K. J. Kirk, and S. Cochran, "1-3 Connectivity lithium niobate composites for high temperature operation," Ultrasonics, vol. 47, no. 1-4, pp. 15-22, 2007.

[59] K. K. Wong, Properties of Lithium Niobate, INSPEC, London, UK, 2002.

[60] G. Ohlendorf, D. Richter, J. Sauerwald, and H. Fritze, "Hightemperature properties of stoichiometric lithium niobate," Diffusion Fundamentals, vol. 8, pp. 6.1-6.7, 2008.

[61] D. S. Hughes, W. L. Pondrom, and R. L. Mims, "Transmission of elastic pulses in metal rods," Physical Review, vol. 75, no. 10, pp. 1552-1556, 1949.

[62] C. K. Jen, L. Piche, and J. F. Bussiere, "Long isotropic buffer rods," Journal of the Acoustical Society of America, vol. 88, no. 1, pp. 23-25, 1990.

[63] A. Sather, "Ultrasonic buffer-rod technique for the hightemperature measurement of the elastic moduli of short specimens," The Journal of the Acoustical Society of America, vol. 43, no. 6, pp. 1291-1294, 1968.

[64] M. W. Grigg, T. J. Davis, A. Cimmino, A. G. Klein, and G. I. Opat, "Commissioning of the Opal reactor elastic moduli of solids - a method suitable for high temperature measurements," Journal of Physics E, vol. 19, no. 12, pp. 10591063, 1986.

[65] S. V. Sinogeikin, D. L. Lakshtanov, J. D. Nicholas, J. M. Jackson, and J. D. Bass, "High temperature elasticity measurements on oxides by Brillouin spectroscopy with resistive and IR laser heating," Journal of the European Ceramic Society, vol. 25, no. 8, pp. 1313-1324, 2005.
[66] F. Ren, E. D. Case, J. R. Sootsman et al., "The high-temperature elastic moduli of polycrystalline $\mathrm{PbTe}$ measured by resonant ultrasound spectroscopy," Acta Materialia, vol. 56, no. 20, pp. 5954-5963, 2008.

[67] O. L. Anderson and D. G. Isaak, "Elastic constants of mantle minerals at high temperature," in Mineral Physics and Crystallography: A Handbook of Physical Constants, T. J. Ahrens, Ed., pp. 64-97, American Geophysical Union, Washington, DC, USA, 1995.

[68] J. Maynard, "Resonant ultrasound spectroscopy," Physics Today, vol. 49, no. 1, pp. 26-31, 1996.

[69] M. A. McGuire, A. D. Christianson, A. S. Sefat et al., "Phase transitions in LaFeAsO: structural, magnetic, elastic, and transport properties, heat capacity and Mössbauer spectra," Physical Review B, vol. 78, no. 9, Article ID 094517, 10 pages, 2008.

[70] J. R. Gladden, G. Li, R. Adebisi, S. Firdosy, T. Caillat, and V. Ravi, "High-temperature elastic moduli of bulk nanostructured $n$ - and p-type silicon germanium," Physical Review B, vol. 82, no. 4, Article ID 045209, 6 pages, 2010.

[71] L. Ekstrom and J. P. Dismukes, "Precipitation of phosphorus from solid solution in Ge-Si alloy," Journal of Physics and Chemistry of Solids, vol. 27, no. 5, pp. 857-863, 1966. 


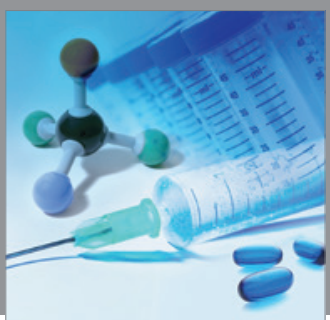

International Journal of

Medicinal Chemistry

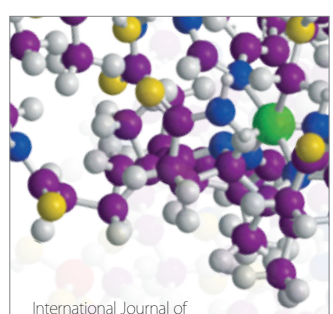

Carbohydrate Chemistry

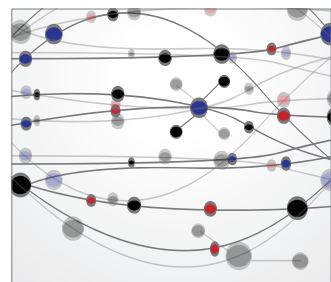

The Scientific World Journal
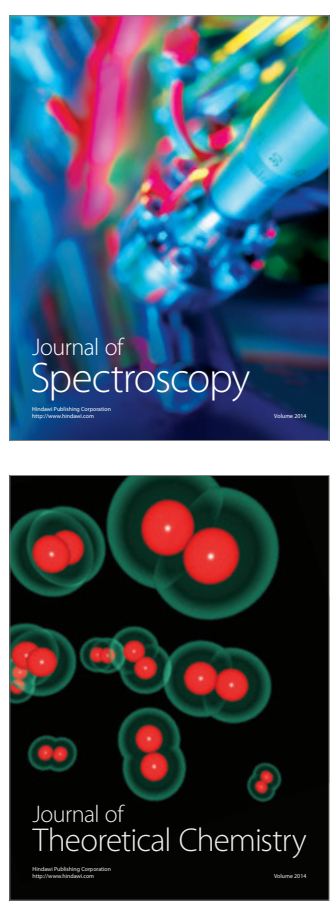
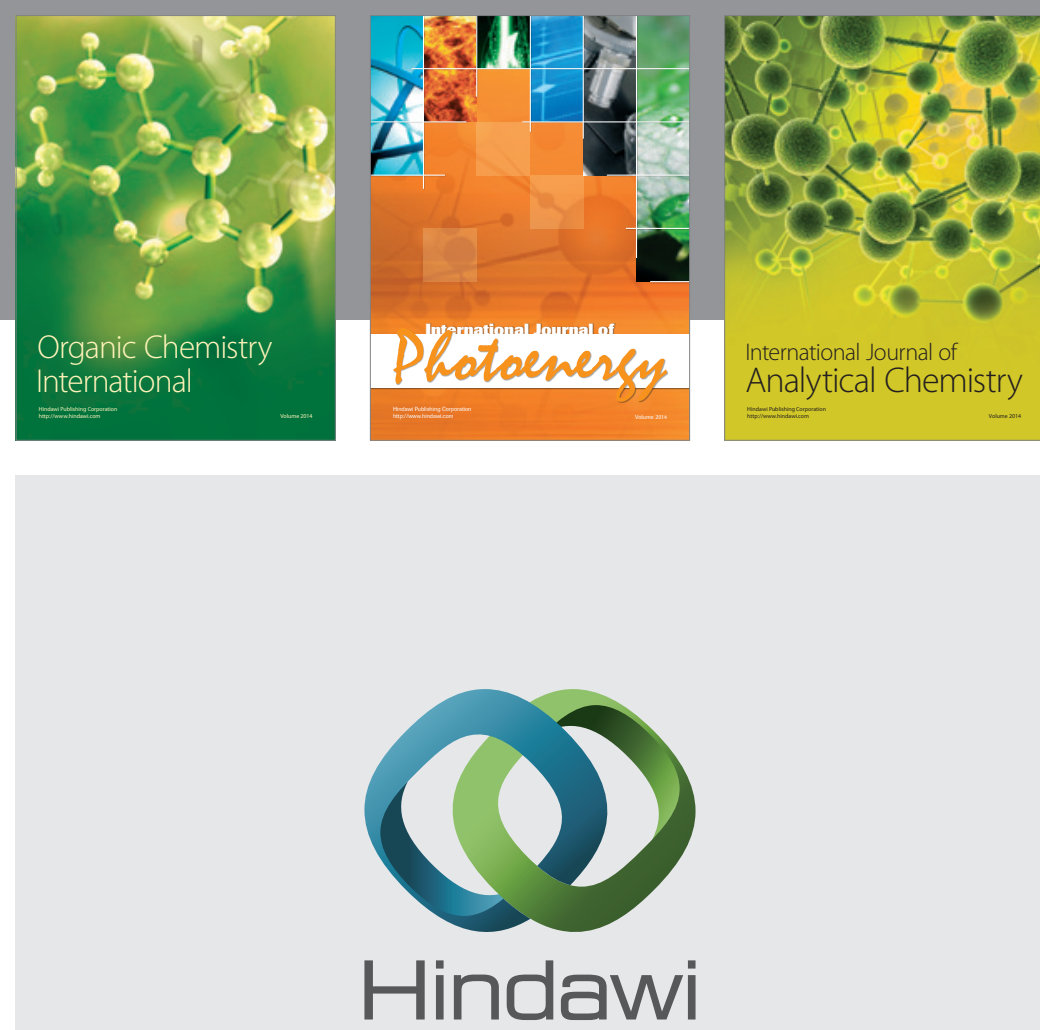

Submit your manuscripts at

http://www.hindawi.com
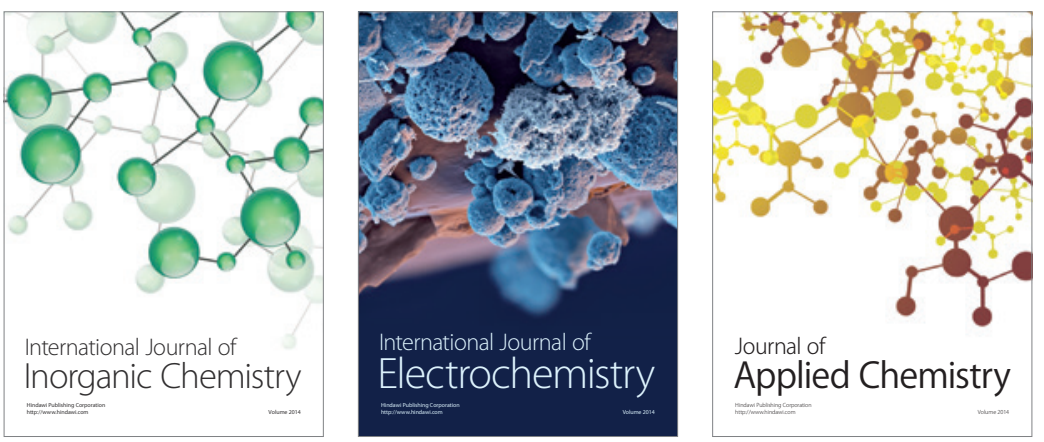

Journal of

Applied Chemistry
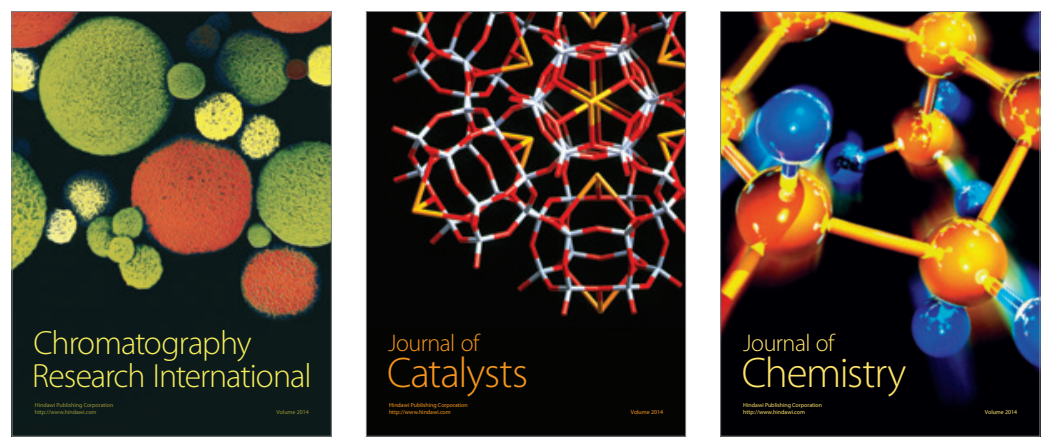
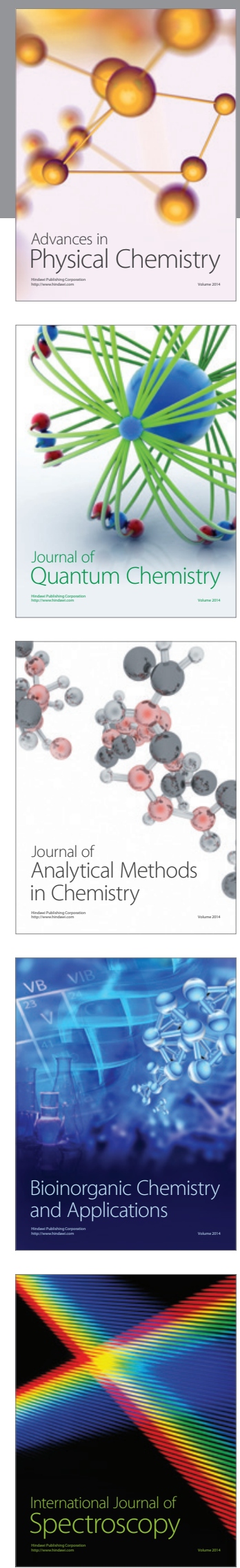